\title{
Information Entropy of Zero-Point Oscillations of a Harmonic Oscillator
}

\author{
A.V. Stepanov ${ }^{1}$, M.A. Stepanov ${ }^{2}$ \\ ${ }^{1}$ National Ozone Monitoring Research and Educational Centre, Belarusian State University, Kurchatov str. 7-816, \\ 220045 Minsk, Belarus \\ ${ }^{2}$ Department of Technical Physics. Information Technologies and Robotics Faculty, Belarusian National Technical \\ University, B. Khmelnitsky str. 9, Building 11, 220013 Minsk, Belarus \\ Email: stepav54@mail.ru
}

\begin{abstract}
Information entropy of zero-point oscillations of a harmonic oscillator is calculated. Its value is expressed in both nats and energy units. It is shown the entropy's physical meaning is determined by the wave particle duality of electromagnetic radiation. The energy resulting from information entropy of initial sub conformation of a myoglobin molecule is derived.
\end{abstract}

Keywords: Thermal equilibrium radiation, molecule, harmonic oscillator, zero-point oscillations, information entropy, myoglobin.

\section{Introduction}

According to Nernst heat theorem, there should be a simple association between entropy and zero-point oscillations. For certain systems their entropy at absolute zero will be exactly zero [1]. Nevertheless, recent studies revealed that it is possible for a molecule to transfer its zero energy into molecular tunnelling one [2]. Therefore, the purpose of this paper is to calculate information entropy of zero-point oscillations of a harmonic oscillator and to determine the entropy's physical meaning.

Like two-faced Janus, harmonic oscillator can be used to describe both thermal equilibrium radiation and a molecule [3]. Thermal radiation is dual by its very nature. It can be considered as a wave, i.e., a continuous process. On the other hand, it can be thought as a stream of photons. Planck used this dualism to get proper expression for zero energy of harmonic oscillator. He assumed a molecule absorbs thermal radiation continuously but emits it discretely [4].So, the expression for information entropy for zero-point oscillations should have presumably two terms responsible for dualism of electromagnetic radiation.

\section{Calculation of Information Entropy of Zero-Point Oscillations}

It was shown that thermodynamic entropy is equal to information one assuming that $k$ is Boltzmann constant in Shannon's formula [5]. Then information entropy at absolute zero, from Nernst heat theorem, should also be zero, which is not. Atomic zero-point oscillations occur at a temperature slightly higher than absolute zero when all thermal oscillations disappear. Its value is given by the expression

$$
T_{0}=\frac{h v}{2 k},
$$

where $h$ and $k$ are Planck and Boltzmann constants, $v$ is the frequency of a harmonic oscillator. Information entropy of low-temperature plateau for molecular tunnelling can be written as

$$
S_{i}^{m, \mu}=k[(\mu+1) \ln (1+\mu)-\mu \ln \mu]
$$

where $m$ is the number of identical quantum oscillators constituting the activation barrier, $\mu$ is the parameter describing the part of oscillator's zero energy used for molecular tunnelling process [2]. Obviously, this part cannot exceed the value of zero energy itself. Substituting $\mu=1 / 2$ into Eq. (2) we get the expression for information entropy in nuce 


$$
\begin{aligned}
& S_{i}^{m, \mu}=k\left[\left(\frac{1}{2}+1\right) \ln \left(1+\frac{1}{2}\right)-\frac{1}{2} \ln \frac{1}{2}\right]=k\left[\frac{3}{2} \ln \frac{3}{2}-\frac{1}{2} \ln \frac{1}{2}\right]= \\
& =k[0.608198-(-0.346574)]=0.954771 k .
\end{aligned}
$$

In Eq. (3) information entropy is measured in energy units. Hence its value in nats is 0.954771 nat.

As noted above, there should be at least two terms in the expression for information entropy responsible for a wave nature and a particle nature of electromagnetic radiation. It can be easily seen there are two terms in Eq. (3), namely 0.608198 nat and 0.346574 nat. Let us clarify their meaning.

\section{$3 \quad$ Physical Meaning of the Entropy under Consideration}

Consider a myoglobin molecule that occupies a certain volume and interacts with thermal equilibrium radiation [5]. Their interaction can be presented by means of a harmonic oscillator. The oscillator's zeropoint oscillations define the radiation's energy mean-square fluctuations in this volume. The fluctuations values are

$$
\begin{aligned}
& \overline{\varepsilon_{\text {quant }}^{2}}=2.09415 \times 10^{-53} \mathrm{erg}^{2}, \\
& \overline{\varepsilon_{\text {int }}^{2}}=1.21875 \times 10^{-53} \mathrm{erg}^{2},
\end{aligned}
$$

The first value is thermal radiation fluctuations that are due to photons motion. The second one arises from the wave nature of radiation. Their ratio

$$
\frac{\overline{\varepsilon_{\text {quant }}^{2}}}{\overline{\varepsilon_{\text {int }}^{2}}}=\frac{2.09415 \times 10^{-53} \mathrm{erg}^{2}}{1.21875 \times 10^{-53} \mathrm{erg}^{2}}=1.71828
$$

can be compared with that followed from Eq. (3)

$$
0.608198 / 0.346574=1.75489 \text {. }
$$

The values of the two ratios are in fairly good agreement. So we can conclude that 0.608198 nat is determined by the particle nature of thermal equilibrium radiation, while 0.346574 nat results from the wave one.

\section{Initial Sub Conformation Energy of a Myoglobin Molecule}

Let us dwell on practical implementation of the derived results. A hypothesis for creation of activation barrier was put forward earlier [3]. The creation process was imagined as a sequence of identical quantum oscillators, each oscillator representing a molecule's sub conformation. More and more sub conformations appear as temperature rises. From the temperature of final sub conformation's creation, the generation of the barrier is complete and a unimolecular reaction starts. It is possible then to measure its constant experimentally. The temperature of initial sub conformation $T_{0}$ can be calculated using Eq. (1). At this temperature the formation of the activation barrier begins. The energy resulting from information entropy of initial sub conformation of a molecule $S_{i}^{m, \mu=1 / 2} \times T_{0}$ can be derived from Eqs. (1) and (3).

Low-temperature fluctuations of a myoglobin molecule were studied experimentally [6]. Three activation barriers $a, b$ and $c$ were observed. Their frequencies $v$ are shown in Table 1 . The two just mentioned barriers characteristics $T_{0}$ and $S_{i}^{m, \mu=1 / 2} \times T_{0}$ are also given there.

Table 1. Characteristics of activation barriers for low-temperature fluctuations of a myoglobin molecule

\begin{tabular}{llll}
\hline Barrier & $a$ & $b$ & $c$ \\
\hline$v(\mathrm{~Hz})$ & $2 \times 10^{6}$ & $1 \times 10^{7}$ & $5 \times 10^{6}$ \\
$T_{0}(\mathrm{~K})$ & $4.8 \times 10^{-5}$ & $2.4 \times 10^{-4}$ & $1.2 \times 10^{-4}$ \\
$S_{\mathrm{i}}^{\mathrm{m}, \mathrm{l}=1 / 2} \times T_{0}(\mathrm{~J})$ & $6.32547 \times 10^{-28}$ & $3.16324 \times 10^{-27}$ & $1.58136 \times 10^{-27}$ \\
\hline
\end{tabular}


As expected the values of $T_{0}$ are very low. However, they are experimentally accessible [7]. So it is possible to explore the initial sub conformation of a myoglobin molecule by experimentation.

\section{Conclusions}

The results of this report can be summarized as follows:

1. Information entropy of zero-point oscillations of a harmonic oscillator is equal to 0.954771 nat, or $0.954771 \times k$, where $k$ is Boltzmann constant.

2. Interaction of a molecule with thermal equilibrium radiation can be described by means of a quantum harmonic oscillator. Its zero-point oscillations define the radiation's energy meansquare fluctuations for this molecule.

3. There are two terms in information entropy, since electromagnetic radiation is inherently dual. 0.608198 nat is determined by the radiation's particle nature, while 0.346574 nat results from the wave one.

4. The energy resulting from information entropy of initial sub conformation of a myoglobin molecule has been derived.

The suggested approach can be used to study zero-point oscillations of vacuum. Several researchers believe these oscillations are made up from vortices of quantum nature [8].

\section{References}

1. W. Nernst, "Uber die Beziehung Zwischen Warmeentwicklung und maximaler Arbeit bei Kondensierten Systemen", Ber. Kgl. Pr. Akad. Wiss., no 52, pp. 933-940, 1906.

2. A. V. Stepanov, M. A. Stepanov, "Informational Entropy of Molecular Tunneling", Proceedings 2018, no 2, pp.151-162. Doi:10.3390/ecea-4-05-005.

3. A.V. Stepanov, "Modeling of metamaterials: a globular protein as a metamaterial prototype for electromagneticacoustic conversion at low temperatures", Proceedings of SPIE, vol. 8070, pp. 807013-1 - 807013-13, 2011.

4. M. Planck, "Uber die Begrundung des Gesetzes der schwarzen Strahlung", Ann. Phys., vol. 342, pp. 642-656, 1912. https://doi.org/10.1002/andp.19123420403.

5. A.V. Stepanov, "Information Entropy of Activation Process: Application for Low-Temperature Fluctuations of a myoglobin molecule", International Journal of Modern Physics B, vol. 29, pp. 1550016-1 - 1550016-18, 2015.

6. D.T. Leeson, "Exploring protein energy landscapes", Ph. D. Thesis, Univ. of Groningen, Netherlands, 1997.

7. S. Truppe, H. J. Williams, M. Hambach, L. Caldwell, N. J. Fitch, E. A. Hinds, B.E. Sauer, and M.R. Tarbutt, "Molecules cooled below the Doppler limit", Nature Physics, vol.13, pp.1173-1176, 2017.

8. L. B. Boldyreva, "Exchange Interaction of Quantum Entities as Interaction of Spin Vortices Created by Quantum Entities in the Physical Vacuum", International Journal of Physics, vol.6, no 57, pp.57-63, 2018. 